Arq. Bras. Med. Vet. Zootec., v.70, n.6, p.1911-1915, 2018

\title{
Hidrocefalia congênita bilateral total em felino: relato de caso
}

[Total Congenital Bilateral Hydrocephalus in Feline: case report]

\author{
C.P. Marinho ${ }^{1}$, F.B. Bacha ${ }^{2}$, G.A. Santos ${ }^{2}$, P.H. Braz ${ }^{3 *}$ \\ ${ }^{1}$ Aluna de pós-graduação - Universidade Federal de Mato Grosso do Sul - Campo Grande, MS \\ ${ }^{2}$ Centro Universitário da Grande Dourados - Dourados, MS \\ ${ }^{3}$ Instituto Federal de Educação, Ciência e Tecnologia Farroupilha - Frederico Westphalen, RS.
}

\begin{abstract}
RESUMO
O objetivo deste trabalho foi relatar um caso raro de hidrocefalia em um felino doméstico, da raça Persa, de 30 dias de idade. $\mathrm{O}$ animal foi atendido com histórico de impossibilidade de manter-se em estação, incoordenação motora, inabilidade para se alimentar sozinho e sustentar o peso da cabeça. No exame clínico, foi observada presença de fontanela aberta, aumento de calota craniana, ataxia, estrabismo unilateral e secreção ocular. A realização de ultrassonografia do crânio levou à confirmação do diagnóstico de hidrocefalia. O paciente permaneceu internado, sendo o protocolo de tratamento empregado constituído de corticosteroide, diurético e protetor gástrico em alta dose, além de alimentação por via oral e fluidoterapia. O animal veio a óbito após 24 horas, sendo encaminhado para realização de necropsia.
\end{abstract}

Palavras-chave: fontanela, ultrassonografia, líquor, neurologia

\begin{abstract}
The objective of this work was to report a rare case of hydrocephalus in a domestic Persian feline, 30 days old. The animal was attended with a history of inability to keep in season, incoordination of the motor, inability to feed itself and support the weight of the head. In the clinical examination, the presence of an open fontanelle, an increase in the skull cap, ataxia, unilateral strabismus and ocular secretion were observed. The ultrasound examination of the skull led to confirmation of the diagnosis of hydrocephalus. The patient remained hospitalized, and the treatment protocol consisted of a corticosteroid, diuretic and gastric protector in high dose, besides oral feeding and fluid therapy. The animal died after 24 hours, being referred for necropsy.
\end{abstract}

Keywords: fontanel, ultrasound, cerebrospinal fluid, neurology

\section{INTRODUÇÃO}

O termo hidrocefalia é comumente utilizado para indicar aumento de volume dos ventrículos cerebrais, particularmente os ventrículos laterais (Chrisman et al., 2005). A doença é caracterizada pelo aumento do acúmulo de líquido cefalorraquidiano (LCR) no crânio, podendo ocorrer em qualquer animal, com maior frequência em filhotes, embora considerada uma congenicidade rara em felinos (Hoski et al.,1993).

$\mathrm{Na}$ forma congênita, o animal nasce com sinais clínicos evidentes para a doença, tais como: estrabismo ventrolateral bilateral, alterações de comportamento e nível de consciência, andar em círculos, crises convulsivas, cegueira cortical e aumento do tamanho do crânio com fontanelas abertas (Lorenz et al., 2011). Já na forma adquirida, normalmente é secundária a alguma doença que cause obstrução total ou parcial do líquor, como neoplasias, traumatismo, edemas cerebrais ou agentes infecciosos que causem encefalite ou meningite (Oliver et al., 1997).

O diagnóstico é baseado no histórico, exame clínico, exame ultrassonográfico, tomografia ou ressonância magnética do crânio (Dewey 2008; Lorenz et al., 2011). A hidrocefalia é visualizada

Recebido em 3 de novembro de 2017

Aceito em 5 de junho de 2018

*Autor para correspondência (corresponding author)

E-mail: paulo.braz@iffaroupilha.edu.br 
na ultrassonografia transcraniana como uma acentuada quantidade de conteúdo anecogênico, líquido homogêneo, aumento dos ventrículos, parênquima cerebral com atrofia e hipoecogênico e comunicação entre os ventrículos devido aos seus aumentos (Carvalho et al., 2007). Entre os métodos de diagnóstico por imagem, o exame ultrassonográfico é o menos oneroso e não invasivo, sendo eficiente no diagnóstico de ventriculomegalia em animais com fontanela aberta.

O tratamento da hidrocefalia é baseado na condição clínica e idade do paciente, podendo ser realizado de forma clínica ou cirúrgica (Dewey, 2008). A forma cirúrgica consiste em promover um desvio do fluxo de LCR do ventrículo lateral para a veia jugular ou cavidade peritoneal (Cage, 1970), enquanto o tratamento medicamentoso é baseado em medicamentos que reduzam a produção do LCR (Dewey, 2008).

O objetivo deste trabalho é relatar um caso raro de um felino doméstico diagnosticado com hidrocefalia bilateral congênita, com base em suas alterações clínicas e anatomopatológicas.

\section{CASUÍSTICA}

Um felino, da raça Persa, de 30 dias de idade, foi atendido apresentando incoordenação motora, incapacidade de se manter em estação, anorexia, emagrecimento e aumento da cabeça em região dorsocranial.

O proprietário relatou que o animal tinha dificuldade para se alimentar desde o nascimento e desenvolvimento motor atrasado, quando comparado aos irmãos nascidos na mesma ninhada.

Ao exame clínico, foi observado aumento de calota craniana (Fig. 1), estrabismo unilateral direito, secreção ocular, mucosa hipocorada, hipotermia e ataxia. O animal apresentava vocalização constante e quedas com rolamento.

Foram solicitados exames complementares, tais como: ultrassonografia do crânio para verificar presença de fontanelas abertas, hemogasometria para dosar $\mathrm{pH}$ sanguíneo e potássio sérico, além de sorologia para toxoplasmose, com o objetivo de pesquisar a causa que levou o animal a desenvolver hidrocefalia congênita.

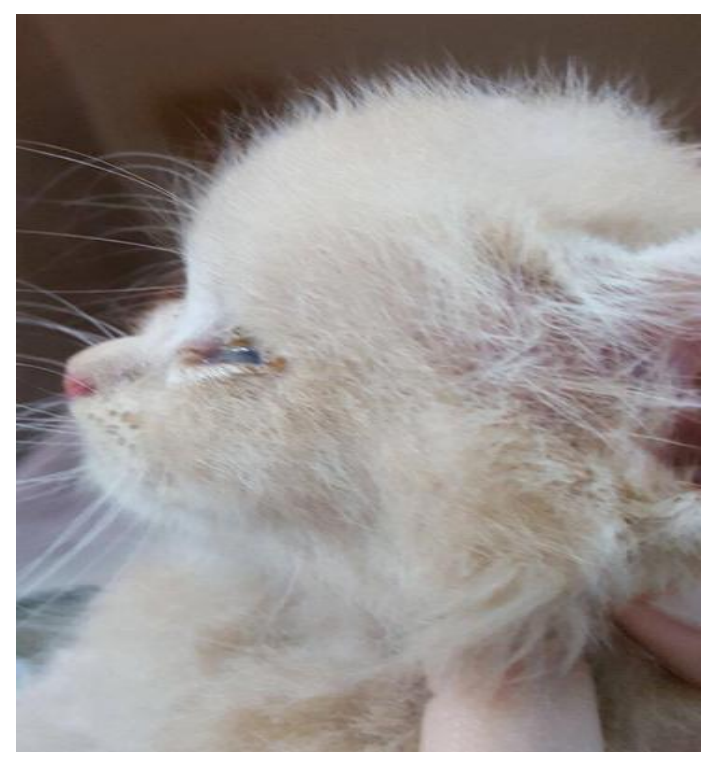

Figura 1. Vista lateral do felino com hidrocefalia. Nota-se aumento da calota craniana frontalmente.

\section{RESULTADOS}

$\mathrm{Na}$ ultrassonografia, foi possível visualizar fontanela frontal aberta, bem como fontanela aberta bilateralmente entre os ossos parietal e temporal, com acúmulo anormal de fluido cerebroespinhal, sendo esses achados compatíveis com hidrocefalia bilateral total (Fig. 2).

O exame de hemogasometria apresentou normalidade, e a sorologia para toxoplasmose mostrou um resultado negativo. Foi solicitada a internação do paciente e realizada venóclise. Institui-se como terapia solução fisiológica $0,9 \%$ $(70 \mathrm{~mL} / \mathrm{kg} / \mathrm{dia})$, dexametasona $(1 \mathrm{mg} / \mathrm{kg} \quad \mathrm{sid})$, omeprazol $(10 \mathrm{mg} / \mathrm{kg}$ sid) e furosemida $(1 \mathrm{mg} / \mathrm{kg}$ bid), além de alimentação por via oral, com leite próprio para felinos. 

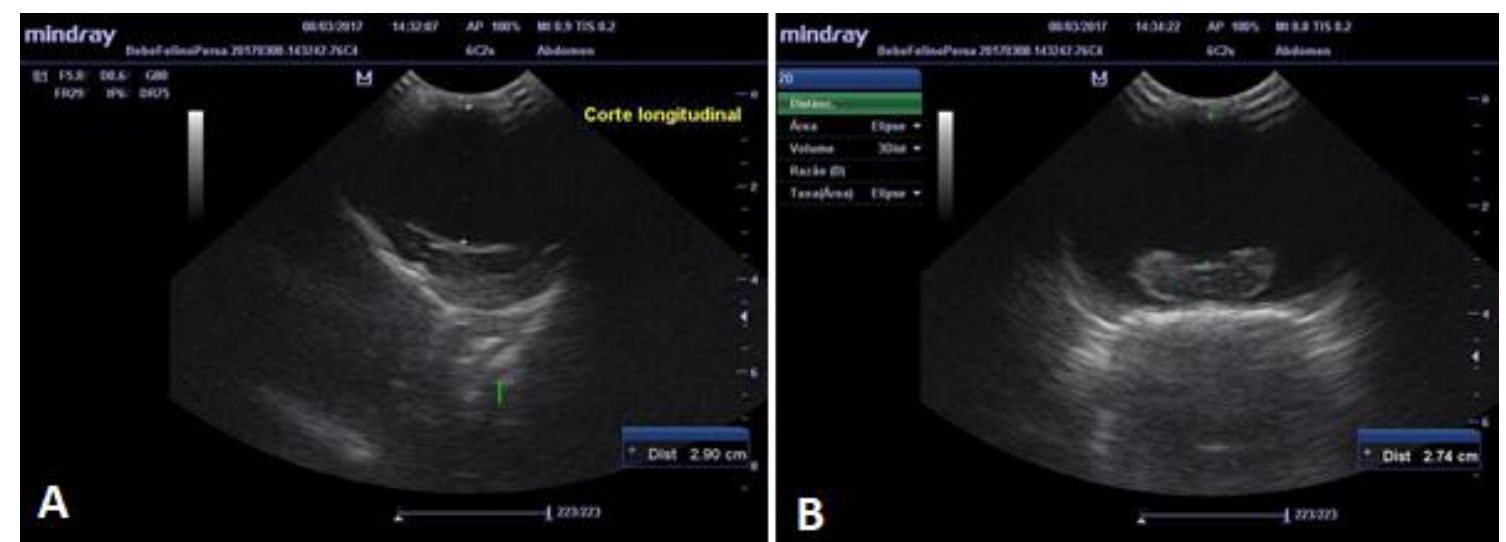

Figura 2. Ultrassonografia transcraniana. (A) Plano de corte longitudinal; nota-se acentuado acúmulo de conteúdo anecogênico no sistema ventricular (ventriculomegalia), medindo $2,90 \mathrm{~cm}$ de altura, e atrofia severa do parênquima encefálico. (B) Plano de corte transversal caudal; visualiza-se comunicação interventricular, com acentuado acúmulo de conteúdo anecogênico (ventriculomegalia), medindo $2,74 \mathrm{~cm}$ de altura, e atrofia severa do parênquima encefálico.

O animal veio a óbito 24 horas após a internação e foi encaminhado para a realização de necropsia. $\mathrm{Na}$ análise macroscópica, foi possível visualizar afinamento e aumento do osso do crânio, alargamento das fontanelas, e os hemisférios cerebrais reduziram-se a uma fina camada de tecido nervoso, desde a região do córtex frontal até a occipital, formando um saco contendo grande quantidade de líquido cefalorraquidiano (Fig. 3).
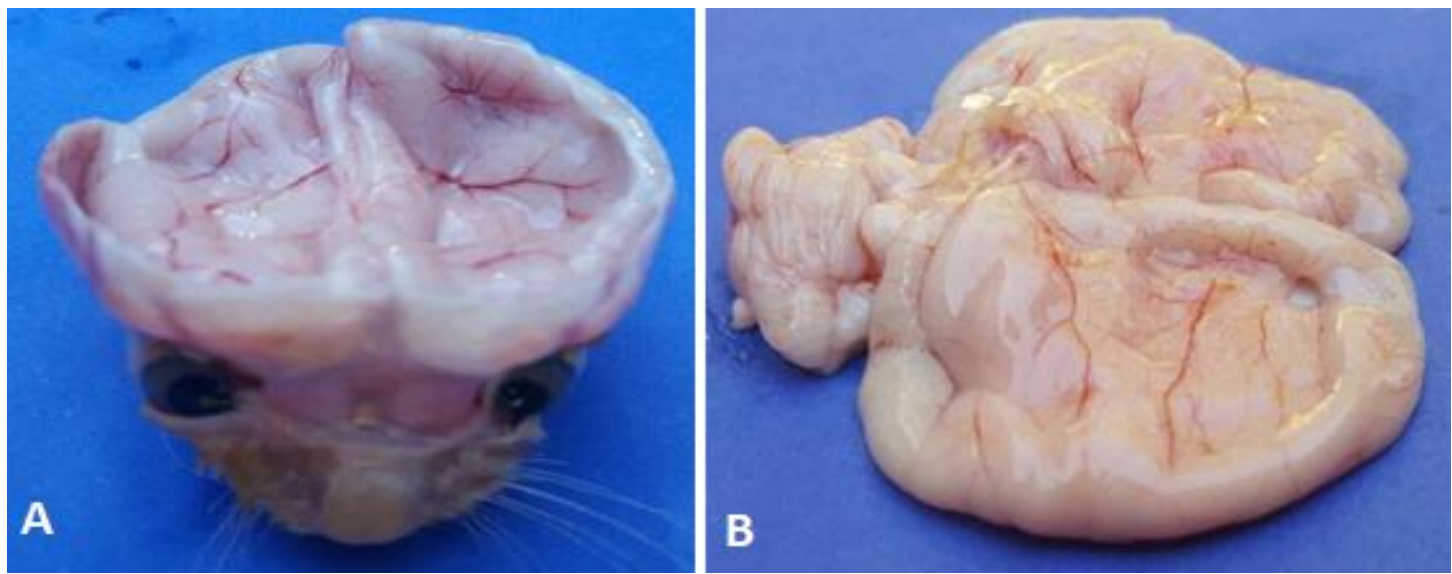

Figura 3. Fotomacrografias de necropsia em um gato com hidrocefalia. Tecido nervoso reduzido a uma fina camada dentro (A) e fora da calota craniana (B), após extravasamento do líquido cefalorraquidiano.

Nos achados histopatológicos do sistema nervoso central (córtex telencefálico), foi possível observar células ependimárias, com alteração de formato (achatadas) e com a substância branca comprimida (Fig. 4). A técnica de coloração empregada foi coloração com hematoxilina e eosina (HE), utilizando-se aumentos de 40x e 10x. Não foram observadas alterações histológicas significativas em outros órgãos. O resultado de necropsia confirmou o diagnóstico de hidrocefalia no felino. 


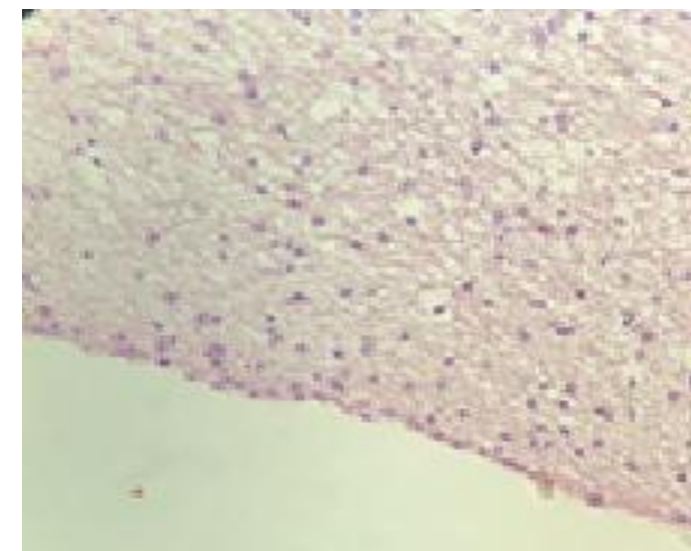

A

Figura 4. Fotomicrografias histopatológicas em um gato com hidrocefalia. (A) Células ependimárias achatadas (aumento 40x) e (B) substância branca comprimida (aumento 10x).

\section{DISCUSSÃO}

A hidrocefalia é uma disfunção envolvendo a absorção e a eliminação do líquido cefalorraquidiano, o que gera acúmulo de LCR intracranial (Ettinger e Feldman, 1997). Ocorre com maior frequência em animais filhotes, em sua forma congênita. Em cães, tem maior ocorrência em raças de porte pequeno, como Poodle, Yorkshire e Chiuaua, porém, em gatos, é considerada uma afecção rara (Hoskins, 1993).

Animais afetados podem apresentar deficiência visual, estrabismo ventrolateral, crises convulsivas, descoordenação, andar em círculos, depressão, atraso no crescimento, fontanelas abertas e aumento no volume do crânio (Hoskins,1993;Ettinger e Feldman, 1997), achados compatíveis com os descritos no relato, além de apresentarem vocalização constante, quedas com rolamento e incapacidade de sustentar a cabeça. Não foram apresentados episódios de crises convulsivas ou andar em círculos.

A ultrassonografia transcraniana, realizada através de fontanelas abertas, é uma boa alternativa, de fácil acesso e baixo custo, para diagnosticar alterações encefálicas estruturais e morfológicas. Outras opções para o diagnóstico de hidrocefalia seriam exames de imagem avançados, como tomografia computadorizada e ressonância magnética. A doença também pode ser diagnosticada mediante a realização de exame radiográfico do crânio, onde é possível observar a cavidade craniana opaca contendo fluido, com aumento da calota craniana e fontanelas ainda abertas (Oliver et al., 1997). Em ultrassonografia do crânio realizada, o animal foi diagnosticado com hidrocefalia bilateral total (Carvalho et al., 2007; Biel et al., 2013).

A hidrocefalia, em sua forma congênita, está relacionada a fatores genéticos e ambientais (Oliver et al., 1997). Em gatos, a forma congênita está associada a uma forma anormal do crânio, podendo ser originada de causas genéticas por uma transmissão autossômica recessiva ou por fatores ambientais, como danos causados no cérebro durante o nascimento, além de infecções perinatais por toxoplasma (Simpson, 1989). Em razão de o animal ter sido negativo sorologicamente à toxoplasmose e de não ter ocorrido nenhum problema no parto, a consanguinidade se tornou um potencial fator que levou à doença, visto que os progenitores do filhote eram irmãos.

O tratamento foi realizado com a utilização de corticosteroide dexametasona, furosemida e omeprazol. O omeprazol, quando testado experimentalmente em cães, provou diminuir a pressão intracraniana devido à diminuição de produção de líquido cefalorraquidiano, a qual pode ocorrer por cerca de $26 \%$ em estudos experimentais em modelo canino utilizando cães saudáveis sem pressão intracraniana aumentada (Javaheri et al., 1997). Não existem atualmente estudos relacionando o uso do omeprazol com a redução do líquido cefalorraquidiano em felinos. 
O tratamento da hidrocefalia exige cuidados gerais de apoio e a utilização de medicamentos que diminuam a produção do líquido cefalorraquidiano, para, assim, reduzir a pressão intracraniana. Dentre esses medicamentos, também se utilizam os diuréticos e os corticosteroides com o mesmo objetivo (Dewey et al., 2008; Kim et al., 2009). Porém, o animal chegou em estado avançado e veio a óbito em 24 horas após ser internado.

Foi realizada a necropsia do animal, o qual apresentou crânio marcadamente aumentado de tamanho, devido ao aumento de diâmetro do cérebro, com $19,47 \mathrm{~cm}$, bem como presença de dilatação acentuada dos ventrículos laterais, com afinamento do córtex telencefálico. Os ventrículos eram preenchidos por líquido límpido e incolor, que extravasa a abertura do crânio, levando a um abaulamento do córtex cerebral, o que confirmou a hidrocefalia bilateral total.

O prognóstico em casos de hidrocefalia é geralmente caracterizado como reservado a ruim. Em casos de hidrocefalia congênita, mesmo tendo um diagnóstico precoce, o prognóstico é considerado reservado. Porém, o diagnóstico e o tratamento, quando instituídos precocemente, podem proporcionar um melhor prognóstico ao animal. (Schoen, 2006).

\section{CONCLUSÕES}

A hidrocefalia é uma afecção rara em felinos, sendo mais comumente encontrada na forma congênita. A realização de ultrassonografia transcraniana é essencial para o diagnóstico. O tratamento se baseia na redução da pressão intracraniana pela diminuição do líquido cefalorraquidiano, além de terapia de suporte para melhora de condições gerais de saúde, porém é uma afecção com um prognóstico ruim a reservado.

\section{REFERÊNCIAS}

BIEL, M.; KRAMER, M.; FORTERRE, F. et al. Outcome of ventriculoperitoneal shunt implantation for treatment of congenital internal hydrocephalus in dogs and cats: 36 cases (20012009). J. Am. Vet. Med. Assoc., v.242, p.948958, 2013.
CAGE, E.D. Surgical treatment of canine hydrocephalus. J. Am. Vet. Med., v.157, p.17291723, 1970.

CARVALHO, C.F.; ANDRADE NETO, J.P.; JIMENEZ C.D. et al. Ultra-sonografa transcraniana em cães com distúrbios neurológicos de origem central. Arq. Bras. Med. Vet. Zootec., v.59, 2007. p.1412-1416.

CHRISMAN, C.; MARIANI, C.; PLATT, S. et al. Demência, estupor e coma. In: Neurologia para o clínico de pequenos animais. São Paulo: Roca, 2005. p.41-82.

DEWEY C.W. Encephalopathies: disorders of the brain. In: DEWEY, C.W.; COSTA, R.C. A pratical guide to canine and feline neurology. 2.ed. Iowa: Wiley-Blackwell, 2008. p.126-129.

ETTINGER, S.J.; FELDMAN, E.C. Tratado de medicina interna veterinária. 4.ed. São Paulo: Manole, 1997.

HOSKINS, J.D. Pediatria veterinária: cães e gatos até seis meses de idade. São Paulo: Manole, 1993. 605p.

JAVAHERI, S.; CORBETT, W.S.; SIMBARTL, L.A. et al. Different effects of omeprazole and Sch 28080 on canine cerebrospinal fluid production. Brain Res. Rev., v.754, p.321-324, 1997.

KIM, J.H.; JEON, H.W.; WOO, E.J.; PARK, H.M. Dilation of the olfactory bulb cavity concurrent with hydrocephalus in four small breed dogs. J. Vet. Sci., v.10, p.173-175, 2009.

LORENZ, M.D.; COATES, J.R.; KENT, M. Stupor or coma. In: MICHAEL, D.; LORENZ, B.S.; DACVIM, D.V.M. Handbook of veterinary neurology. 5.ed. Philadelphia: W.B. Saunders, 2011. p.346-383.

OLIVER, Jr, J.E.; LORENZ, M.D.; KORNEGAY, J.N. In. Handbook of veterinary neurology. Philadelphia: W.B. Saunders, 1997. p.274-312.

SCHOEN, A. Acupuntura veterinária: da arte antiga à medicina moderna. 2.ed. São Paulo: Roca, 2006. 603p.

SIMPSON, S.T. neurology. In: KIRK, R.W. (Ed.). Currente veterinary therapy $X$. Philadelphia: WB Saunders, 1989. p.842-846. 